\title{
Computation of Antieigenvalues of Bounded Linear Operators Via Centre of Mass
}

\author{
Kallol Paul • Gopal Das · Lokenath Debnath
}

Published online: 25 October 2014

(C) Springer India Pvt. Ltd. 2014

\begin{abstract}
We introduce the concept of $\theta$-antieigenvalue and $\theta$-antieigenvector of a bounded linear operator on complex Hilbert space. We study the relation between $\theta$-antieigenvalue and centre of mass of a bounded linear operator and compute antieigenvalue using the relation. This follows the notion of symmetric antieigenvalues introduced by Hossein et al. (J. Math. Anal. Appl. 70:3877-3884, 2004). We show that the concept of real antieigenvalue, imaginary antieigenvalue and symmetric antieigenvalue follows as a special case of $\theta$-antieigenvalue. We also show how the concept of total antieigenvalue is related to the $\theta$-antieigenvalue. In fact, we show that all the concepts of antieigenvalues studied so far follows from the concept of $\theta$ - antieigenvalue. We illustrate with example how to calculate the $\theta$-antieigenvalue for an operator acting on a finite dimensional Hilbert space.
\end{abstract}

Keywords Antieigenvalue $\cdot$ Antieigenvectors $\cdot$ Bounded linear operator · Centre of mass

Mathematics Subject Classification $47 \mathrm{~B} 44 \cdot 47 \mathrm{~A} 63 \cdot 47 \mathrm{~B} 15$

\section{Introduction}

The concept of angle of an operator was introduced by Gustafson [5-7] in 1967 while studying the problems in perturbation theory of semi-group generators. For a bounded linear operator

K. Paul ( $\varangle)$

Department of Mathematics, Jadavpur University, Kolkata 700032, India

e-mail: kalloldada@gmail.com

G. Das

Department of Mathematics, Jalpaiguri Govt. Engg. College, Jalpaiguri 735102, West Bengal, India e-mail: gopaldasju@gmail.com

L. Debnath

Department of Mathematics, The University of Texas-Pan American, 1201 West University Drive,

Edinburg, TX 78539, USA

e-mail: debnathl@utpa.edu 
$T$ on a complex Hilbert space $H$ with the norm $\|$.$\| and the inner product \langle$,$\rangle the cosine of$ the angle of the operator $T$ is defined as

$$
\cos \Phi(T)=\inf _{T f \neq 0} \frac{\operatorname{Re}\langle T f, f\rangle}{\|T f\|\|f\|} .
$$

The similar concept was also introduced by Krei $\breve{n}$ [20] in 1969 which he called the deviation of $T$ and denoted by $\operatorname{dev} . T$. The $\cos \Phi(T)$ has another interpretation as first antieigenvalue ( real antieigenvalue) $\mu_{1}(T)$ of $\mathrm{T}$ which was also introduced by Gustafson [8]:

$$
\mu_{1}(T)=\inf _{T f \neq 0} \frac{\operatorname{Re}\langle T f, f\rangle}{\|T f\|\|f\|} .
$$

The vector $\mathrm{f}$ for which $\mu_{1}(T)$ is attained (if exists), is called antieigenvector. The higher antieigenvalues are defined as follows :

$$
\mu_{n}(T)=\inf _{T f \neq 0}\left\{\frac{\operatorname{Re}\langle T f, f\rangle}{\|T f\|\|f\|}, \quad f \perp\left\{f^{(1)}, f^{(2)}, f^{(3)}, \ldots, f^{(n-1)}\right\}\right\}
$$

where $f^{(k)}$ is the $k$ th antieigenvector of $T$.

The first antieigenvalue can be interpreted as the cosine of the largest angle (real) through which any vector can be rotated by the action of $T$. The concept of antieigenvalues is studied by Gustafson [8-12], Gustafson and Rao [13,14], Gustafson and Seddighin [15], Das et al. [2] ,Paul [22], Paul and Das [23].

Likewise Gustafson also introduced the concept of imaginary antieigenvalue as

$$
\inf _{T f \neq 0} \frac{\operatorname{Im}\langle T f, f\rangle}{\|T f\|\|f\|} .
$$

The total cosine of an operator $T$ is defined as

$$
|\cos | T=\inf _{T f \neq 0} \frac{|\langle T f, f\rangle|}{\|T f\|\|f\|}
$$

The concept of total antieigenvalues is studied by Gustafson and Seddighin [16], Seddighin [24], Hossein et al. [18]. In [19], first author Paul et.al. introduced the concept of symmetric antieigenvalue and antieigenvector for an operator $T$ as follows:-

$$
\Phi_{T}(f)=\frac{\operatorname{Re}\langle T f, f\rangle+\operatorname{Im}\langle T f, f\rangle}{\sqrt{2}\|T f\|\|f\|} \quad T f \neq 0
$$

and

$$
\cos \Phi_{S}(T)=\inf _{T f \neq 0} \Phi_{T}(f) .
$$

The symmetric antieigenvalue is also denoted by $\mu_{S}$. The vector $f$ for which $\Phi_{T}(f)$ attains the minimum (if exists ) is called the symmetric antieigenvector of $T$. For a selfadjoint operator $T$ with the eigenvalues $\lambda_{1} \geq \lambda_{2} \geq \lambda_{3} \geq \cdots \geq \lambda_{n}, \cos \Phi(T)=\frac{\sqrt{\lambda_{1} \lambda_{n}}}{\lambda_{1}+\lambda_{n}}$, whereas $\cos \Phi(i T)=0$ [according to definition of Gustafson] rather abruptly, although $i T$ has the eigenvalues $i \lambda_{1}, i \lambda_{2}, i \lambda_{3}, \ldots, i \lambda_{n}$. But the symmetric antieigenvalues of both $T$ and $i T$ are same i.e $\mu_{S}(T)=\mu_{S}(i T)$ for a self adjoint operator T. The definition of the antieigenvalue involves only real part of numerical range $W(T)$ of an operator $T$, but the symmetric antieigenvalue depends upon both real and imaginary part of the numerical range $W(T)$ of $T$. Following our concept of symmetric antieigenvalue Gustafson and Seddighin 
[17] studied slant antieigenvalues and slant antieigenvectors of operators, in which they did not show any explicit relation between slant antieigenvalue and total antieigenvalue.

In [23], Paul and Das proved Min-max equality of a bounded linear operator $T$ on a complex Hilbert space $H$ using the concept of orthogonality of bounded linear operators in the sense of James [3] and studied the relation between centre of mass and antieigenvalues. The Min-max equality in operator trigonometry goes as follows:

Min-max equality: For a bounded linear opeartor $T$ on a complex Hilbert space

$$
\sup _{\|x\|=1} \inf _{\epsilon \in R}\|(\epsilon T-I) x\|^{2}=\inf _{\epsilon \in R} \sup _{\|x\|=1}\|(\epsilon T-I) x\|^{2}
$$

The Min-max equality in operator trigonometry was obtained by Gustafson [4] in 1968, Asplund and Pták [1] in 1971. In [23] using the concept of orthogonality of operators in the sense of James [3] we proved that

For a bounded linear opeartor $T$ on a complex Hilbert space

$$
\sup _{\|x\|=1} \inf _{\lambda \in C}\|(\lambda T-I) x\|^{2}=\inf _{\lambda \in C} \sup _{\|x\|=1}\|(\lambda T-I) x\|^{2}
$$

In [23] we introduced the concept of real centre of mass and total centre of mass of a bounded linear operator and studied their relation with antieigenvalues. We here mention the definitions of the real centre of mass and total centre of mass for the sake of completeness of information.

Definition 1 For any two bounded linear operators $T$ and $A$, there exists scalars $\epsilon_{0} \in R$ and $\lambda_{0} \in C$ such that

$$
\left\|T-\epsilon_{0} A\right\| \leq\|T-\epsilon A\| \quad \forall \epsilon \in R
$$

and

$$
\left\|T-\lambda_{0} A\right\| \leq\|T-\lambda A\| \quad \forall \lambda \in C
$$

Then numbers $\epsilon_{0}$ and $\lambda_{0}$ are called real centre of mass of $T$ with respect to $A$ and total centre of mass of $T$ with respect to $A$ respectively.

We here introduce the concept of $\theta$-antieigenvalue and explore the relation between $\theta$ antieigenvalue and centre of mass of an operator. The concept of antieigenvalue, imaginary antieigenvalue and symmetric antieigenvalue then follows as a special case of $\theta$ antieigenvalue. We also show how the concept of total antieigenvalue is related to the concept of $\theta$ - antieigenvalue. Finally we give examples of matrices to calculate $\theta$-antieigenvalue.

\section{$\theta$-Antieigenvalues}

Definition 2 ( $\theta$-antieigenvalue) Let $T$ be a bounded linear operator on a complex Hilbert space $H$ and $\theta \in R$. Define

$$
\mu_{\theta}(f)=\frac{\cos \theta \operatorname{Re}\langle T f, f\rangle+\sin \theta \operatorname{Im}\langle T f, f\rangle}{\|T f\|\|f\|}, \quad T f \neq \theta
$$

and

$$
\mu_{\theta}(T)=\inf _{T f \neq 0} \frac{\cos \theta \operatorname{Re}\langle T f, f\rangle+\sin \theta \operatorname{Im}\langle T f, f\rangle}{\|T f\|\|f\|}
$$

$\mu_{\theta}(T)$ is called $\theta$-antieigenvalue of $T$ and the vectors $f$ for which $\mu_{\theta}(T)$ attains the infimum (if exsits) are called $\theta$-antieigenvectors of $T$. 
If $\theta=0$ then we get first antieigenvalue (real antieigenvalue) as

$$
\mu_{0}(T)=\inf _{T f \neq 0} \frac{\operatorname{Re}\langle T f, f\rangle}{\|T f\|\|f\|}=\cos T .
$$

If $\theta=\frac{\pi}{2}$ then we get imaginary antieigenvalue as

$$
\mu_{\frac{\pi}{2}}(T)=\inf _{T f \neq 0} \frac{\operatorname{Im}\langle T f, f\rangle}{\|T f\|\|f\|}
$$

and if $\theta=\frac{\pi}{4}$ then we get symmetric antieigenvalue

$$
\mu_{\frac{\pi}{4}}(T)=\inf _{T f \neq 0} \frac{\operatorname{Re}\langle T f, f\rangle+\operatorname{Im}\langle T f, f\rangle}{\sqrt{2}\|T f\|\|f\|}=\mu_{s}(T) .
$$

We have studied the antieigenvectors in $[2,18,19,22]$ using the concept of stationary vectors, the definition of which is given below:

\section{Definition 3 Stationary vector.}

Let $\phi(f)$ be a functional of a unit vector $\mathrm{f} \in \mathrm{H}$. Then $\phi(f)$ is said to have a stationary value at $\mathrm{f}$ if the function $w_{g}(t)$ of a real variable $\mathrm{t}$, defined as

$$
w_{g}(t)=\phi\left(\frac{f+t g}{\|f+t g\|}\right)
$$

has a stationary value at $\mathrm{t}=0$ for any arbitrary but fixed vector $\mathrm{g} \in \mathrm{H}$. The vector $\mathrm{f}$ is then called a stationary vector.

We write

$$
\Phi(f)=\frac{\cos \theta \operatorname{Re}\langle T f, f\rangle+\sin \theta \operatorname{Im}\langle T f, f\rangle}{\|T f\|\|f\|} ; \quad f \in H, T f \neq 0 .
$$

and find the necessary and sufficient condition for a unit vector $\mathrm{f}$ to be a stationary vector of $\Phi(f)$. For this we define

$$
w_{g}(t)=\frac{(\cos \theta \operatorname{Re}\langle T(f+t g),(f+t g)\rangle+\sin \theta \operatorname{Im}\langle T(f+t g),(f+t g)\rangle)^{2}}{\|T(f+t g)\|^{2}\|f+t g\|^{2}}
$$

where $\mathrm{g}$ is an arbitrary but fixed vector of $\mathrm{H}$.

If $\mathrm{f}$ is a stationary vector then we have $w_{g}^{\prime}(0)=0$ and so we get

$$
2\|T f\|^{2}(\cos \theta A f+\sin \theta B f)-(a \cos \theta+b \sin \theta)\left(T^{*} T f+\|T f\|^{2} f\right)=0,
$$

where $A=\operatorname{ReT}, B=\operatorname{Im} T, a=\operatorname{Re}\langle T f, f\rangle, b=\operatorname{Im}\langle T f, f\rangle$.

This is the necessary and sufficient condition for $\Phi(f)$ to be stationary at a vector $\mathrm{f}$. Thus we obtain the following theorem

Theorem 1 Let $f$ be a unit $\theta$-antieigenvector of a bounded linear operator $T$. Then $f$ satisfies the following equation

$$
2\|T f\|^{2}(\cos \theta A f+\sin \theta B f)-(a \cos \theta+b \sin \theta)\left(T^{*} T f+\|T f\|^{2} f\right)=0,
$$

where $A=\operatorname{Re} T, B=\operatorname{Im} T, a=\operatorname{Re}\langle T f, f\rangle, b=\operatorname{Im}\langle T f, f\rangle$.

Remark 1 Putting $\theta=0, \frac{\pi}{2}$, and $\frac{\pi}{4}$ we get the characteristic equation for $\mathrm{f}$ to be a real antieigenvector, imaginary antieigenvector and symmetric antieigenvector respectively. 
As we have studied in $[2,18,19]$ we can find $\theta$-antieigenvalues for bounded selfadjoint operators and normal operators and obtain similar results. We here find the relation between centre of mass and $\theta$-antieigenvalue via Min-max equality. We first state the following theorem the proof of which is given in [23] using the concept of orthogonality of bounded linear operators.

Theorem 2 For bounded linear operators $T$ and $\lambda I$, where $\lambda \in C$ and $|\lambda|=1$, on a Hilbert space $H$

$$
\sup _{\|f\|=1} \inf _{\epsilon \in R}\|(\epsilon T-\lambda I) f\|^{2}=\inf _{\epsilon \in R} \sup _{\|f\|=1}\|(\epsilon T-\lambda I) f\|^{2}
$$

Now we prove the following theorem.

Theorem 3 Let $\epsilon_{0}$ be a real centre of mass of $T$ with respect to the operator $\lambda I$ [where $\lambda=\cos \theta+i \sin \theta, \quad \theta \in R]$ and $T$ is a bounded linear operator on a complex Hilbert space $H$. Then

$$
\mu_{\theta}(T)=\lim _{n \rightarrow \infty} \frac{\cos \theta \operatorname{Re}\left\langle T f_{n}, f_{n}\right\rangle+\sin \theta \operatorname{Im}\left\langle T f_{n}, f_{n}\right\rangle}{\left\|T f_{n}\right\|\left\|f_{n}\right\|}
$$

where $\left\{f_{n}\right\}$ is a sequence of unit vectors in complex Hilbert space $H$ such that $\operatorname{Re}\langle(\lambda I-$ $\left.\left.\epsilon_{0} T\right) f_{n}, T f_{n}\right\rangle \rightarrow 0$ and $\left\|\left(\lambda I-\epsilon_{0} T\right) f_{n}\right\| \rightarrow\left\|\lambda I-\epsilon_{0} T\right\|$.

Proof Since $\epsilon_{0}$ is a real centre of mass of $T$ with respect to $\lambda I$, we obtain a sequence of unit vectors $\left\{f_{n}\right\}$ in $H$ such that $\operatorname{Re}\left\langle\left(\lambda I-\epsilon_{0} T\right) f_{n}, T f_{n}\right\rangle \rightarrow 0$ and $\left\|\left(\lambda I-\epsilon_{0} T\right) f_{n}\right\| \rightarrow\left\|\lambda I-\epsilon_{0} T\right\|$.

Now

$$
\begin{aligned}
\left\|\lambda I-\epsilon_{0} T\right\| & =\inf _{\epsilon \in R}\|\lambda I-\epsilon T\| \\
& =\inf _{\epsilon \in R} \sup _{\|f\|=1}\|(\lambda I-\epsilon T) f\|
\end{aligned}
$$

Using the min-max theorem we obtain

$$
\begin{aligned}
\left\|\lambda I-\epsilon_{0} T\right\|^{2} & =\lim _{n \rightarrow \infty}\left\{1-\left(\frac{\cos \theta \operatorname{Re}\left\langle T f_{n}, f_{n}\right\rangle+\sin \theta \operatorname{Im}\left\langle T f_{n}, f_{n}\right\rangle}{\left\|T f_{n}\right\|}\right)^{2}\right\} \\
& \leq \sup _{\|f\|=1}\left\{1-\left(\frac{\cos \theta \operatorname{Re}\langle T f, f\rangle+\sin \theta \operatorname{Im}\langle T f, f\rangle}{\|T f\|}\right)^{2}\right\} \\
& \leq \sup _{\|f\|=1} \inf _{\epsilon \in R}\|(\lambda I-\epsilon T) f\|^{2} \\
& =\left\|\lambda I-\epsilon_{0} T\right\|^{2}
\end{aligned}
$$

Therefore

$$
\begin{aligned}
\inf _{\|f\|=1} \frac{\cos \theta \operatorname{Re}\langle T f, f\rangle+\sin \theta \operatorname{Im}\langle T f, f\rangle}{\|T f\|} & =\lim _{n \rightarrow \infty} \frac{\cos \theta \operatorname{Re}\left\langle T f_{n}, f_{n}\right\rangle+\sin \theta \operatorname{Im}\left\langle T f_{n}, f_{n}\right\rangle}{\left\|T f_{n}\right\|} \\
& =\mu_{\theta}(T) .
\end{aligned}
$$

This completes the theorem. 


\section{Total Antieigenvalue and $\boldsymbol{\theta}$-Antieigenvalue}

We here show the relation between total antieigenvalue and $\theta$-antieigenvalue.

Lemma 1 Let $f \in H, T f \neq 0$ and $\theta \in R$. Then

$$
\sup _{\theta \in R} \mu_{\theta}(f)=\sup _{\theta \in R} \frac{\cos \theta \operatorname{Re}\langle T f, f\rangle+\sin \theta \operatorname{Im}\langle T f, f\rangle}{\|T f\|\|f\|}=\frac{|(T f, f)|}{\|T f\|\|f\|} .
$$

Proof For a fixed $\mathrm{f}$ we can think of $\mu_{\theta}(f)$ as a function from $\mathrm{R}$ to $\mathrm{R}$. Let $\Psi: R \longrightarrow R$ be defined as

$$
\Psi(\theta)=\frac{\cos \theta \operatorname{Re}\langle T f, f\rangle+\sin \theta \operatorname{Im}\langle T f, f\rangle}{\|T f\|\|f\|} .
$$

Then using elementary calculus we see that $\Psi$ attains its maximum at $\cos \theta=\frac{\operatorname{Re}\langle T f, f\rangle}{|\langle T f, f\rangle|}$ and

$$
\sup _{\theta \in R} \Psi(\theta)=\frac{|\langle T f, f\rangle|}{\|T f\|\|f\|} .
$$

Theorem $4|\cos | T=\inf _{T f \neq 0} \sup _{\theta \in R} \mu_{\theta}(f)$.

Proof Follows from Lemma 1 as

$$
\sup _{\theta \in R} \Psi(\theta)=\sup _{\theta \in R} \frac{\cos \theta \operatorname{Re}\langle T f, f\rangle+\sin \theta \operatorname{Im}\langle T f, f\rangle}{\|T f\|\|f\|}=\frac{|\langle T f, f\rangle|}{\|T f\|\|f\|}
$$

and so

$$
|\cos | T=\inf _{T f \neq 0} \sup _{\theta \in R} \mu_{\theta}(f)=\inf _{T f \neq 0} \frac{|\langle T f, f\rangle|}{\|T f\|\|f\|} .
$$

Lemma $2 \inf _{\theta \in R} \inf _{\epsilon \in R}\left\|\epsilon e^{i \theta} T-I\right\|=\inf _{\lambda \in C}\|\lambda T-I\|$.

Proof By a result of [21] there exists $\lambda_{0}=\epsilon_{0} e^{i \theta_{0}} \in C$ such that $\left\|\lambda_{0} T-I\right\|=\inf _{\lambda \in C}\|\lambda T-I\|$ and so

$$
\left\|\epsilon_{0} e^{i \theta_{0}} T-I\right\|=\left\|\lambda_{0} T-I\right\| \leq\|\lambda T-I\|=\left\|\epsilon e^{i \theta} T-I\right\| \quad \forall \epsilon \in R \text { and } \theta \in R .
$$

For each $\theta \in R$ there exists $\epsilon(\theta)=\epsilon_{\theta}$ such that

$$
\left\|\epsilon_{\theta} e^{i \theta} T-I\right\| \leq\left\|\epsilon e^{i \theta} T-I\right\| \quad \forall \epsilon .
$$

For $\theta_{0}, \epsilon_{\theta_{0}}$ may or may not be equal to $\epsilon_{0}$ but

$$
\left\|\epsilon_{0} e^{i \theta_{0}} T-I\right\| \leq\left\|\epsilon_{\theta_{0}} e^{i \theta_{0}} T-I\right\| \leq\left\|\epsilon e^{i \theta_{0}} T-I\right\| \forall \epsilon .
$$

We choose

$$
\begin{aligned}
\epsilon(\theta) & =\epsilon_{\theta} \quad \theta \neq \theta_{0} \\
& =\epsilon_{0} \quad \theta=\theta_{0} .
\end{aligned}
$$


Then

$$
\begin{aligned}
& \inf _{\theta \in R} \inf _{\epsilon \in R}\left\|\epsilon e^{i \theta} T-I\right\| \\
= & \inf _{\theta \in R}\left\|\epsilon(\theta) e^{i \theta} T-I\right\| \\
= & \left\|\epsilon_{0} e^{i \theta_{0}} T-I\right\| \\
= & \inf _{\lambda \in C}\|\lambda T-I\| .
\end{aligned}
$$

We next show that

\section{Theorem 5}

$$
\inf _{T f \neq 0} \sup _{\theta \in R} \mu_{\theta}(f)=\sup _{\theta \in R} \inf _{T f \neq 0} \mu_{\theta}(f) .
$$

Proof We have

$$
\cos T=\sqrt{1-\inf _{\epsilon \in R}\|\epsilon T-I\|^{2}} \text { so that } \inf _{\epsilon \in R}\|\epsilon T-I\|^{2}=1-\inf _{T f \neq 0}\left(\frac{\operatorname{Re}\langle T f, f\rangle}{\|T f\|\|f\|}\right)^{2}
$$

and

$$
|\cos | T=\sqrt{1-\inf _{\lambda \in C}\|\lambda T-I\|^{2}} \text { so that } \inf _{\lambda \in C}\|\lambda T-I\|^{2}=1-\inf _{T f \neq 0}\left(\frac{|\langle T f, f\rangle|}{\|T f\|\|f\|}\right)^{2}
$$

Using Lemma 2 we have

$$
\begin{aligned}
& \inf _{\lambda \in C}\|\lambda T-I\|^{2}=\inf _{\theta \in R} \inf _{\epsilon \in R}\left\|\epsilon e^{-i \theta} T-I\right\|^{2} \\
\Rightarrow & \inf _{\lambda \in C}\|\lambda T-I\|^{2}=\inf _{\theta \in R}\left\{1-\inf _{T f \neq 0}\left(\frac{\operatorname{Re}\left\langle e^{-i \theta} T f, f\right\rangle}{\left\|e^{-i \theta} T f\right\|\|f\|}\right)^{2}\right\} \\
\Rightarrow & \inf _{\lambda \in C}\|\lambda T-I\|^{2}=1-\sup _{\theta \in R} \inf _{T f \neq 0} \mu_{\theta}^{2}(f) \\
\Rightarrow & \sup _{\theta \in R} \inf _{T f \neq 0} \mu_{\theta}^{2}(f)=1-\inf _{\lambda \in C}\|\lambda T-I\|^{2} \\
\Rightarrow & \sup _{\theta \in R} \inf _{T f \neq 0} \mu_{\theta}^{2}(f)=|\cos |^{2} T \\
\Rightarrow & \sup _{\theta \in R} \inf _{T f \neq 0} \mu_{\theta}(f)=\inf _{T f \neq 0} \sup _{\theta \in R} \mu_{\theta}(f) .
\end{aligned}
$$

This completes the proof.

Example $T=\left(\begin{array}{cc}2-3 i & 0 \\ 0 & 3+2 i\end{array}\right)$ be an operator on a two dimensional complex Hilbert space H. Let $z=\left(z_{1}, z_{2}\right)^{t} \in H$, where $\left|z_{1}\right|^{2}+\left|z_{2}\right|^{2}=1$. Then, $\operatorname{Re}\left(T z, e^{i \theta} z\right)=3 \cos \theta+$ $2 \sin \theta-(\cos \theta+5 \sin \theta)\left|z_{1}\right|^{2}|(T z, z)|=\sqrt{\frac{26}{4}+26\left(\left|z_{1}\right|^{2}-\frac{1}{2}\right)^{2}}$ and $\|T z\|=\sqrt{13}$. Then $|\cos | T=\frac{1}{\sqrt{2}}$ and the total cosine attains at the unit vector $z=\left(z_{1}, z_{2}\right)^{t}$ where $\left|z_{1}\right|^{2}=\frac{1}{2}$ and the total centre of mass is $\frac{5+i}{26}$.

The $\theta$-antieigenvalue of $T$ is as follows:- 
Case I When $\sin \left(\theta+\tan ^{-1} \frac{1}{5}\right) \leq 0$, then $\mu_{\theta}(T)=\frac{3 \cos \theta+2 \sin \theta}{\sqrt{13}}$ and it attains at the vector $z=\left(z_{1}, z_{2}\right)^{t}$ where $\left|z_{1}\right|=0$ and the centre of mass of $T$ with respect to $e^{i \theta} I$ where $I$ is the identity operator, is $\frac{3 \cos \theta+2 \sin \theta}{13}$

Case II When $\sin \left(\theta+\tan ^{-1} \frac{1}{5}\right)>0$, then $\mu_{\theta}(T)=\frac{2 \cos \theta-3 \sin \theta}{\sqrt{13}}$ and it attains at the vector $z=\left(z_{1}, z_{2}\right)^{t}$ where $\left|z_{1}\right|=1$ and the centre of mass of $T$ with respect to $e^{i \theta} I$ where $I$ is the identity operator, is $\frac{2 \cos \theta-3 \sin \theta}{13}$

From $\theta$-antieigenvalue, we obtain antieigenvalue and symmetric antieigenvalue of $T$. When $\theta=0$, then antieigenvalue $\cos T=\mu_{0}(T)=\frac{2}{\sqrt{13}}$ and the corresponding centre of mass is $\frac{2}{13}$. When $\theta=\frac{\pi}{4}$ then the symmetric antieigenvalue is $\mu_{S}(T)=\mu_{\frac{\pi}{4}}(T)=-\frac{1}{\sqrt{26}}$ and the corresponding centre of mass $\epsilon_{0}=\frac{-1}{13 \sqrt{2}}$.

Also inf $\operatorname{iff}_{f 0} \sup _{\theta \in R} \mu_{\theta}(f)=\sup _{\theta \in R} \inf _{T f \neq 0} \mu_{\theta}(f)=\frac{1}{\sqrt{2}}$.

Acknowledgments We thank Professor T. K. Mukherjee for his invaluable suggestions while preparing this paper.

\section{References}

1. Asplund, E., Pták, V.: A minimax inequality for operators and a related numerical range. Acta. Math. 126, 53-62 (1971)

2. Das, K.C., DasGupta, M., Paul, K.: Structure of the antieigenvectors of a strictly accretive operator International. J. Math. Math. Sci. 21(4), 761-766 (1998)

3. James, R.C.: Orthogonality and linear functionals in normed linear spaces. Trans. Am. Math. Soc. 61, 265-292 (1947)

4. Gustafson, K.: A min-max theorem. Not. Am. Math. Soc. 15, 799 (1968d)

5. Gustafson, K.: Angle of an operator and positive operator products. Bull. Am. Math. Soc. 74, 488-492 (1968a)

6. Gustafson, K.: Positive(noncommutating) operator products and semigroups. Math. Zeit. 105, 160-172 (1968b)

7. Gustafson, K.: A note on left multiplication of semigroup generators. Pac. J. Math. 24, 463-465 (1968c)

8. Gustafson, K.: Antieigenvalue inequalities in operator theory. In: Shisha, O. (ed.) Inequalities III, Proceedings Los Angeles Symposium, 1969, pp. 115-119, Academic Press, New York (1972)

9. Gustafson, K.: An extended operator trigonometry. Linear Algebra Appl. 319, 117-135 (2000)

10. Gustafson, K.: Operator Trigonometry. Linear Multilinear Algebra 37, 139-159 (1994)

11. Gustafson, K.: Matrix trigonometry. Linear Algebra Appl. 217, 117-140 (1995)

12. Gustafson, K.: Interaction antieigenvalues. J. Math. Anal. Appl. 299, 174-185 (2004)

13. Gustafson, K., Rao, D.: Numerical range and accretivity of operator products. J. Math. Anal. Appl. 60, 693-702 (1977)

14. Gustafson, K., Rao, D.: Numerical Range: The Field Values of Linear Operators and Matrices. Springer, New York (1997)

15. Gustafson, K., Seddighin, M.: Antieigenvalue bounds. J. Math. Anal. Appl. 143, 327-340 (1989)

16. Gustafson, K., Seddighin, M.: A note on total antieigenvalues. J. Math. Anal. Appl. 178, 603-611 (1993)

17. Gustafson, K., Seddighin, M.: Slant antieigenvalues and slant antieigenvectors of operators. Linear Algebra Appl. 432, 1348-1362 (2010)

18. Hossein, Sk.M., Das, K.C., Debnath, L., Paul, K.: Bounds for total antieigenvalue of a normal operator International. J. Math. Math. Sci. 70, 3877-3884 (2004)

19. Hossein, Sk.M., Paul, K., Debnath, L., Das, K.C.: Symmetric anti-eigenvalue and symmetric antieigenvector. J. Math. Anal. Appl. 345, 771-776 (2008)

20. Krein̆, H.: Angular localization of the spectrum of a mulplicative integral in a Hilbert space. Funct. Anal. Appl. 3, 89-90 (1969)

21. Paul, K., Hossein, Sk.M., Das, K.C.: Orthogonality on B(H, H) and minimal-norm operator. J. Anal. Appl. 6, 169-178 (2008)

22. Paul, K.: Antieigenvectors of the generalized eigenvalue problem and an operator inequality complementary to Schwarz's inequality. Novi Sad J. Math. 38(2), 25-31 (2008) 
23. Paul, K., Das, G.: Cosine of angle and center of mass of an operator. Mathematica Slovaca 62(1), 109-122 (2012)

24. Seddighin, M.: Antieigenvalues and total antieigenvalues of normal operators. J. Math. Anal. Appl. 274(1), 239-254 (2002) 